\title{
Uso del Kikuyo (Pennisetum Clandestinum L), residuo de la poda de áreas verdes para la obtención de ácido piroleñoso con fines agropecuarios
}

Use of Kikuyo (Pennisetum Clandestinum L), residue from the pruning of green areas for obtaining pyroligneous acid for agricultural purposes

Diego Burbano-Salas ${ }^{I}$

Recibido: 15-07-2019 / Revisado: 25-07-2019 /Aceptado: 20-08-2019/ Publicado: 10-09-2019

\begin{abstract}
DOI: https://doi.org/10.33262/cienciadigital.v3i3.4..882

The large volumes of biomass generated in the maintenance of green areas are considered a focal point for the production of organic waste. In the present work, it is proposed to obtain piroleñoso acid from the precursor Pennisetum Clandestinum L (kikuyo), a very common waste product of pruning and gardening; Describes the thermochemical process for the validation of the technology for the production of the product and utilizable by-products (coal and tar) and the evaluation of the macro elements and the structural components of the substance by means of the Fourier Transform Infrared Spectrometry (FTIR) method. (Fourier transform infrared spectroscopy), by a direct comparison, it is shown that its properties are similar to the product from Bamboo (Phyllostachys pubescense $\mathrm{f}$ ) which has characteristics that can increase root growth in vegetables, in farm animals improves the digestibility of Nutrients and reduces harmful intestinal coliforms. The results obtained describe a yield of between 20 and $30 \%$ for the gases with a maximum delivered energy that oscillates between 150 and $1000 \mathrm{kcal}$.
\end{abstract}

Keywords: pyroligneous acid, biocarbon, pyrolysis, organic waste.

\section{Resumen}

Los grandes volúmenes de biomasa generados en el mantenimiento de áreas verdes se los considera un punto focal de producción de desechos orgánicos. En el presente trabajo, se propone la obtención del ácido piroleñoso a partir del precursor Pennisetum Clandestinum L (kikuyo), residuo muy común producto de las podas y de adecentamiento de jardines; se describe todo el proceso termoquímico de validación de la tecnología para obtención del producto y

\footnotetext{
${ }^{1}$ Facultad de Ciencias, Escuela de Ciencias Químicas, Escuela Superior politécnica de Chimborazo, Riobamba, Ecuador. E-mail: dburbano@espoch.edu.ec
} 
subproductos aprovechables (carbón, alquitrán) y la evaluación de los macro elementos y los componentes estructurales de la sustancia mediante el método de Espectrometría Infrarroja con Transformada de Fourier (FTIR del inglés Fourier transform infrared spectroscopy), por una comparativa directa, se demuestra que sus propiedades son similares al producto proveniente del Bambú (Phyllostachys pubescens $f$ ) el cual posee características que pueden incrementar el crecimiento radicular en vegetales, en animales de granja mejora la digestibilidad de los nutrientes y reduce los coliformes intestinales perjudiciales. Los resultados obtenidos describen un rendimiento de entre 20 y $30 \%$ para los gases con una energía máxima entregada que oscila entre 150 y $1000 \mathrm{kcal}$.

Palabras clave: ácido piroleñoso, biocarbón, pirolisis, Residuos orgánicos.

\section{Introducción}

La crisis mundial con respecto a la contaminación y a la degradación del suelo crece a tasas alarmantes, sobre todo porque las soluciones a estos problemas tienen que ver con actividades antropogénicas que utilizan indiscriminadamente los recursos y generan una cantidad de residuos de todo tipo. Los procesos térmicos a partir de la biomasa son motivo de investigación e implementación de sistemas en varios lugares del mundo científico, pero sobre todo es una necesidad del creciente desarrollo. (Jun, 2006). Ecuador cuenta con una gran cantidad de residuos de biomasa provenientes principalmente de procesos de industrias de alimentos, que están siendo aprovechadas principalmente en la generación alternativa de energía, sin embargo, las tecnologías que se aplican no son lo suficientemente eficientes para competir con las fuentes tradicionales, deteniendo el crecimiento de este tipo de incitativas, por consiguiente, aumentando la cantidad de residuos. (Aguilera, 2006). La sustancia obtenida es un subproducto del pirólisis conocido como vinagre de madera, es generalmente marrón oscuro, viscoso y compuesto por una mezcla muy compleja de hidrocarburos oxigenados. Es útil para el mejoramiento del suelo, la actividad de las semillas, la germinación y especialmente en la actividad biológica de los ataques de hongos y termitas. (Choi, 2009).

La composición y rendimiento de vinagre de madera dependen de las condiciones del proceso y de las composiciones de la biomasa usada. Se han realizado extensos estudios sobre las composiciones químicas y las aplicaciones basadas en materiales de partida con roble, sakura, té verde, bambú, eucalipto, planta de manglar y residuos de agricultura. (CharMarker, 2009). Sin embargo, no ha habido ninguna investigación sistemática en el análisis químico avanzado del vinagre de Pennisetum Clandestinum L (kikuyo). El objetivo de la presente investigación es la caracterización de ácido piroleñoso obtenido a partir de este producto de la poda, con fines de su posible utilización agropecuaria, para lo cual se describe el proceso de producción y se realiza la caracterización de los principios activos del producto obtenido. 


\section{Materiales y Métodos}

\section{Ubicación}

El área seleccionada para la obtención de residuos de kikuyo fue la Escuela Superior Politecnica de Chimborazo (ESPOCH), en la parte externa de la institución se ha adecuado un lugar como botadero de material vegetativo producto de la poda y mantenimiento de las áreas verdes.

\section{Recolección de la materia prima}

El proceso de recolección y separación de agentes inorgánicos como fundas y envolturas no reviste mayor dificultad y estos residuos se depositaron en la basura inorgánica común. El área de descarga a cielo abierto mencionada se encuentra aledaña al almacenamiento de agua potable que abastece al sector y a la institución, detectándose procesos de descomposición, presencia de vectores y contaminación visual.

\section{Toma y procesamiento de la muestra}

Se tomó una muestra de $42 \mathrm{~kg}$ de $P$. clandestinum $L$, del lugar seleccionado el cual se sometió al proceso de pirólisis a una temperatura entre los $200^{\circ} \mathrm{C}-800^{\circ} \mathrm{C}$. (Burnette, An Introduction to Wood Vinager, 2013). Para la caracterización de la materia prima se tomaron daos bibliográficos. En la Tabla 1 se encuentra representada las fracciones de proteína cruda de kikuyo, tanto en su parte de fracción soluble, fracción potencialmente degradable y fracción no degradable, dando a conocer las características proteicas que posee la biomasa utilizada, un $62 \%$ es degradable un $31.2 \%$ fracción soluble y un $12.7 \%$ constituye una parte no degradable. (Hagner, 2013).

Tabla 1:Fracciones de la proteína cruda de muestras de Pennisetum Clandestinum $L$

\begin{tabular}{lccc}
\hline \multicolumn{4}{c}{ Fracciones de la PC } \\
\hline & $\boldsymbol{a}$ & $\boldsymbol{b}$ & $\boldsymbol{C}$ \\
Promedio & 31.2 & 62.5 & 12.7 \\
Máximo & 42.9 & 72.2 & 19.5 \\
Mínimo & 18.9 & 44.9 & 7.07 \\
D. E. & 5.21 & 8.50 & 4.66 \\
C. V., \% & 16.7 & 13.6 & 36.7 \\
\hline
\end{tabular}


$\mathrm{a}=$ fracción soluble $; \mathrm{b}=$ fracción potencialmente degradable; $\mathrm{c}=$ fracción no degradable; D. E. = Desviación Estándar; C.V = coeficiente de variación.

En el método in situ, la fracción $a$ corresponde a la fracción proteica que rápidamente desaparece (NRC 2001). En promedio, el 31.2\% de la PC del pasto kikuyo está representado por la fracción $a$ con una variación que oscila entre el 18.9 y el $42.9 \%$ (Hagner, 2013)

\section{Metodología de producción}

Para la extracción, se utilizó un reactor de lecho fijo a escala piloto, herméticamente cerrado para asegurar condiciones de reacción anaeróbicas, el equipo así dispuesto (figura 3) fue utilizado en el proceso de pirólisis obteniendo varios productos de oxidación, las emisiones de la pirólisis son recogidas por un sistema de destilación con agua de refrigeración a $10^{\circ} \mathrm{C}$; al finalizar el proceso de destilación el líquido es el ácido piroleñoso. (Ikeshima, 1999)

\section{Caracterización del producto obtenido}

De la revisión bibliográfica se presume que dentro del ácido existen más de 200 tipos de componentes químicos involucrados y estos incluyen principalmente ácidos orgánicos, fenólicos, alcanos, compuestos de alcohol y éster. (Ikeshima, 1999). En la evaluación de los macroelementos y componentes estructurales se ha utilizado la metodología de espectrometría infrarroja por transformadas de Fourier (FTIR). Cuando la radiación IR se hace pasar a través de una muestra, parte de la radiación es absorbida y parte pasa a través de la muestra. La señal resultante en el detector es un espectro que representa una 'huella digital' molecular de la muestra. La utilidad de la espectroscopia de infrarrojo surge debido a que diferentes estructuras químicas (moléculas) producen diferentes huellas espectrales. El FTIR utiliza interferómetria para grabar información sobre un material colocado en el haz IR. La transformada de Fourier otorga resultados en los espectros que los analistas pueden utilizar para identificar o cuantificar el material. (Bradley, 2002)

\section{Resultados y Discusión}

\section{Proceso de obtención propuesto}

El ácido piroleñoso es un líquido que se obtiene de la destilación seca de material vegetativo previamente deshidratado, está integrado por un 70 a $90 \%$ de humedad y muchos compuestos orgánicos, entre ellos el ácido acético y el alcohol metilico, en el proceso se obtiene una serie de subproductos aprovechables como son el biocarbón y el alquitrán. 
La figura 1 muestra, el proceso que se realiza para la obtención del ácido piroleñoso, la energía solar interviene en el proceso de secado, como producto de esto se ocasiona emisiones de calor residual, residuos forestales, como la leña que es el pilar para el incremento de la temperatura en la pirólisis, se emiten gases de combustión además de ácidos, fenoles, alcanos que ingresan al proceso de destilación, durante este proceso se genera, alquitrán vegetal, destilado y como residuo final de la pirólisis de la biomasa, se obtiene biocarbón y ceniza producto de la quema de los residuos forestales.

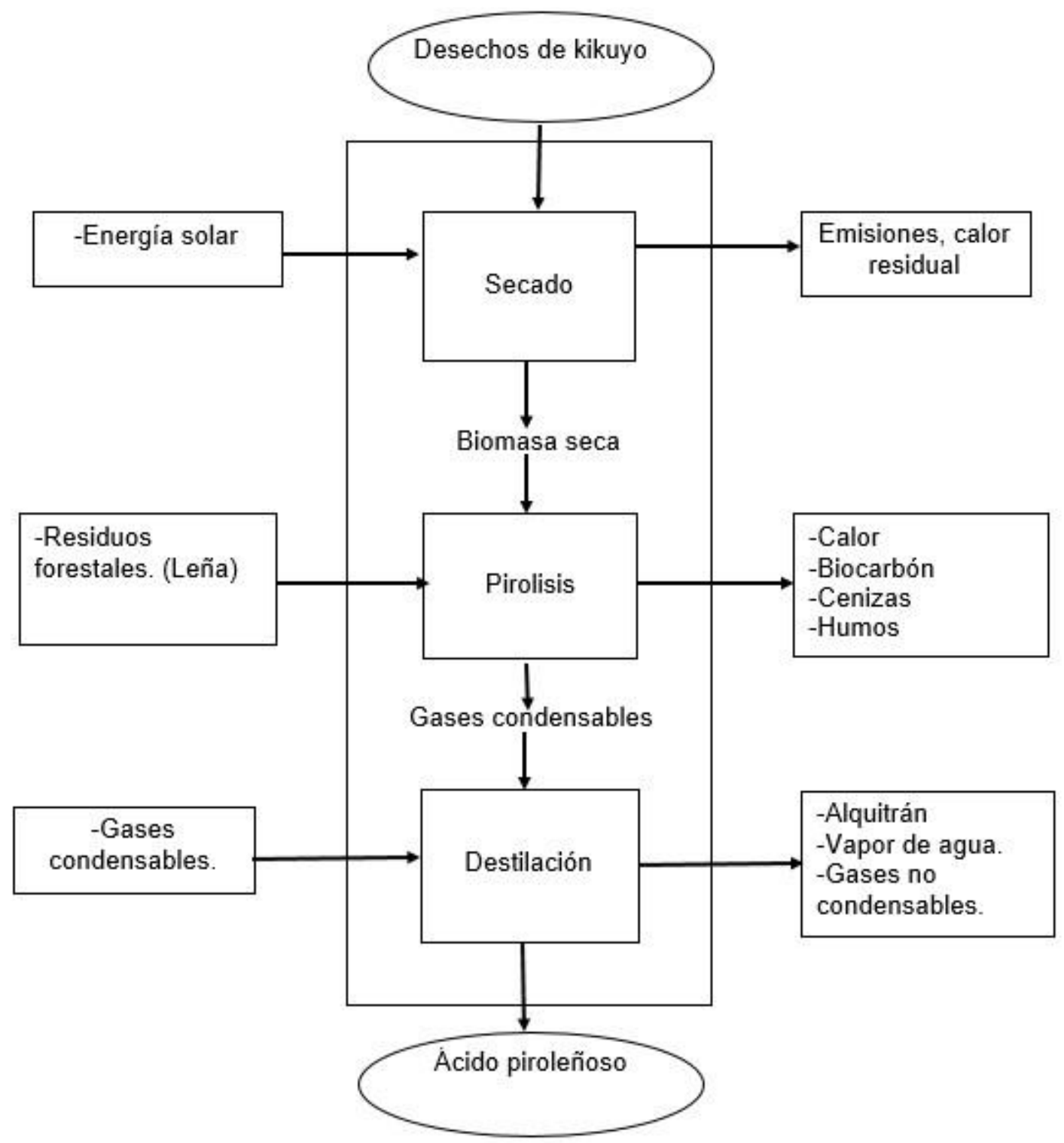

Figura 1: Diagrama de proceso de obtención de ácido piroleñoso.

Partiendo de aproximadamente $70 \mathrm{Kg}$ de biomasa en la cual el $60 \%$ corresponde a la materia prima analizada y reconociendo que el porcentaje de humedad del proceso es 
relativamente bajo, se recupera aproximadamente medio litro de destilado que principalmente contiene ácido acético, como subproductos se obtienen $17 \mathrm{~kg}$ de biocarbón y $95 \mathrm{ml}$ de alquitrán, estimando un rendimiento de entre 20 y $30 \%$ para los gases con una energía máxima entregada que oscila entre 150 y $1000 \mathrm{kcal}$.

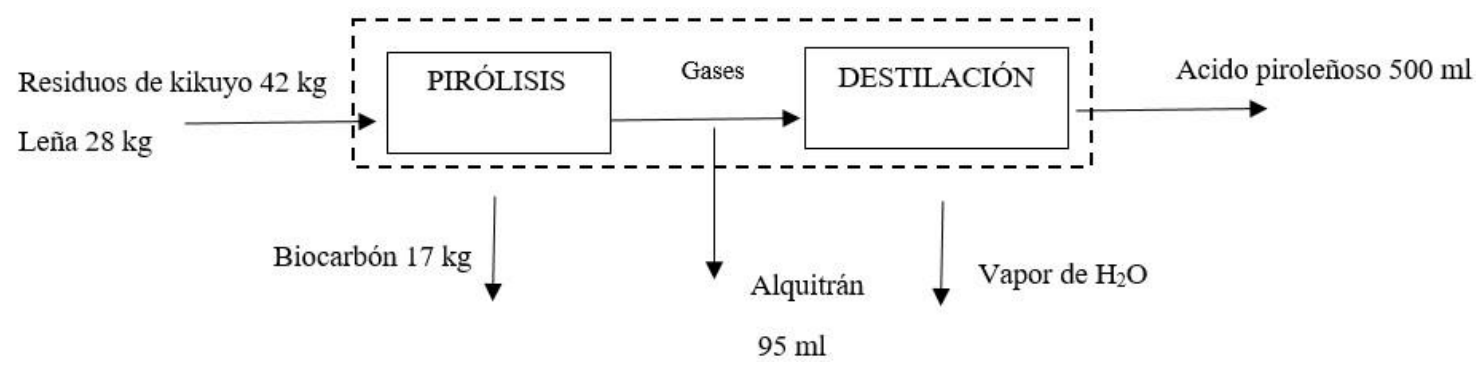

Figura 2. Balance de materia de residuos procesados para la obtención de ácido piroleñoso.

La figura 3 presenta la transformación del desecho por medio de un proceso termoquímico. Los gases obtenidos se someten a procesos de condensación simple. Las temperaturas del proceso oscilan entre 300,450 y $600{ }^{\circ} \mathrm{C}$. Los resultados fundamentales que se obtienen son: Rendimiento a carbón oscila entre 25 y $35 \%$, Rendimiento a gas oscilan entre 21 y $29 \%$, Rendimiento a alquitrán entre 5 y $9 \%$.

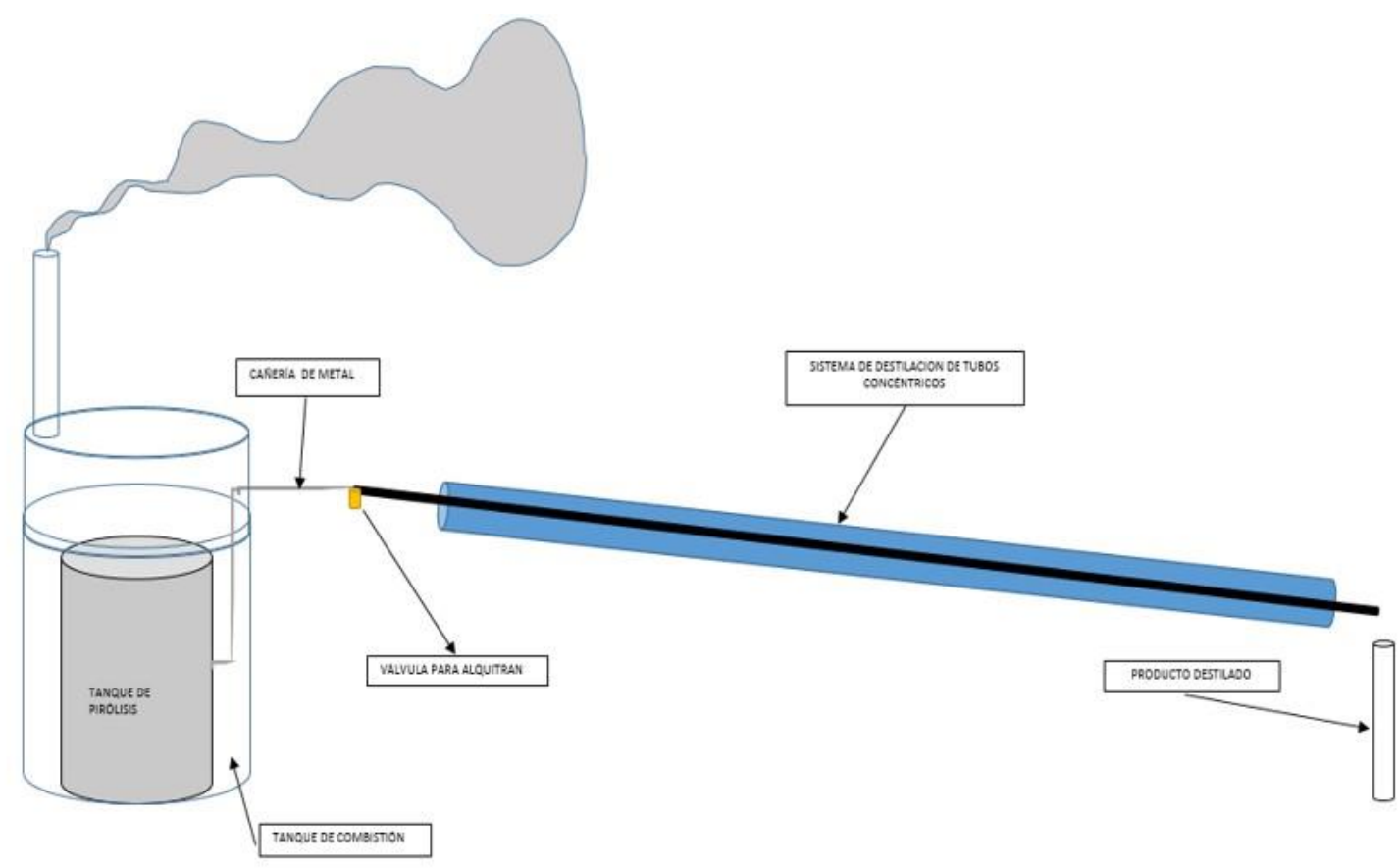

Figura 3. Sistema de destilación de humos producto de la pirólisis. 
La Figura 4 muestra el análisis de la muestra de destilado realizado en un espectrofotómetro infrarrojo utilizando la metodología (FTIR), se realizó una dilución de $15 \mathrm{ml}$ de destilado en $200 \mathrm{ml}$ de agua des ionizada, se observan bandas que reflejan su composición, los compuestos orgánicos se clasificaron en ácidos carboxílicos, fenoles, cetonas y aldehídos que es lo que hace presumibles gases fundamentales como: $\mathrm{H}_{2}, \mathrm{CO}$, $\mathrm{CH}_{4}, \mathrm{CO}_{2}, \mathrm{C}_{2} \mathrm{H}_{4}, \mathrm{C}_{2} \mathrm{H}_{6}, \mathrm{C}_{2} \mathrm{H}_{2}$, entre otros.

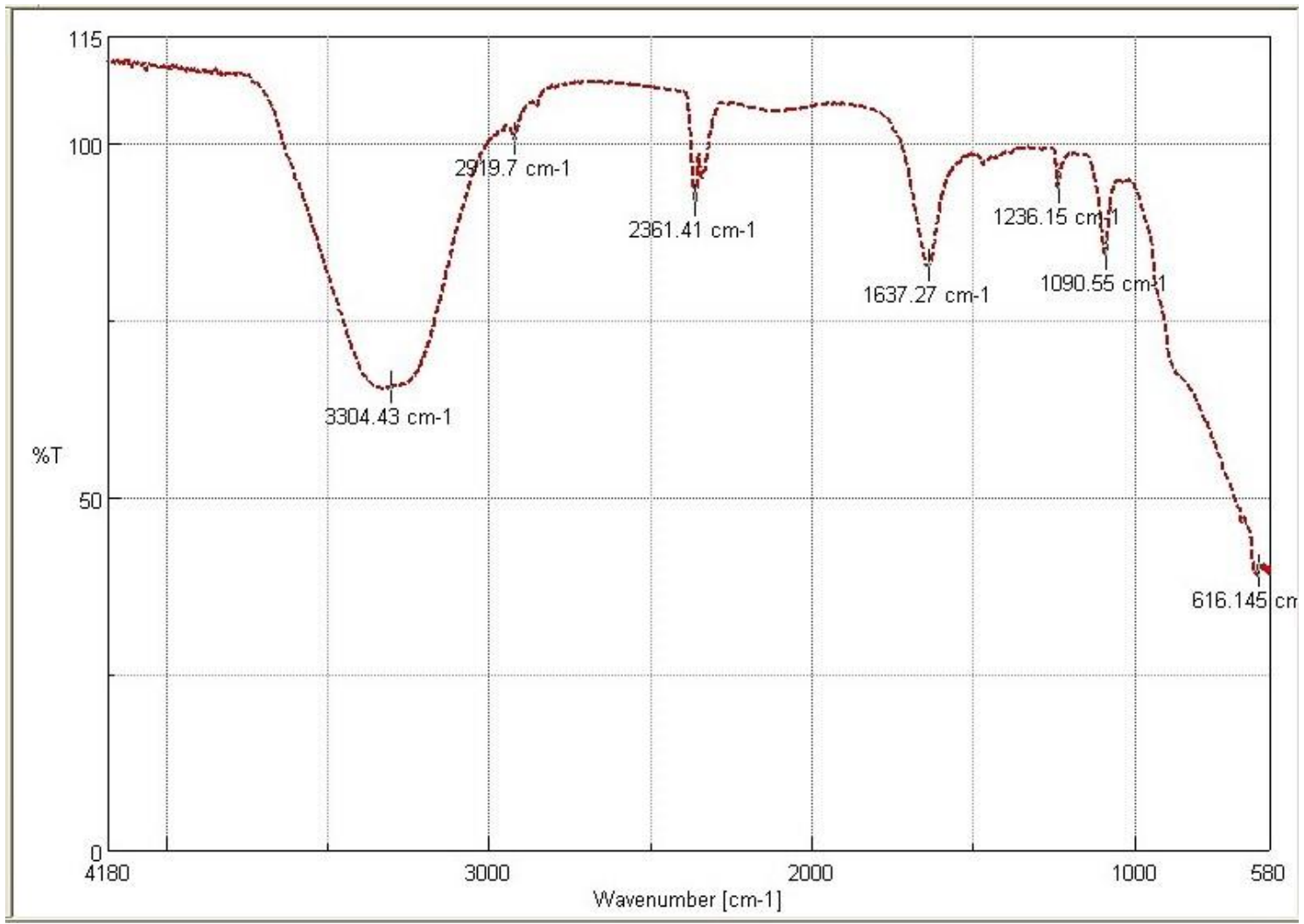

Fuente: (Laboratorio de Química Analítica, ESPOCH et al., 2017)

Figura 4. Análisis en el Espectro IR del "condensado de kikuyo" con longitud de onda.

Tabla 2Composición química e interpretación de resultados del condensado. Análisis en el Espectro IR (Laboratorio de Química Analítica, ESPOCH, 2017).
Longitud de onda. Rango de longitud Grupo y clase
Compuestos
$\mathbf{c m}^{-1}$ de onda. $\mathrm{cm}^{-1}$
presentes 


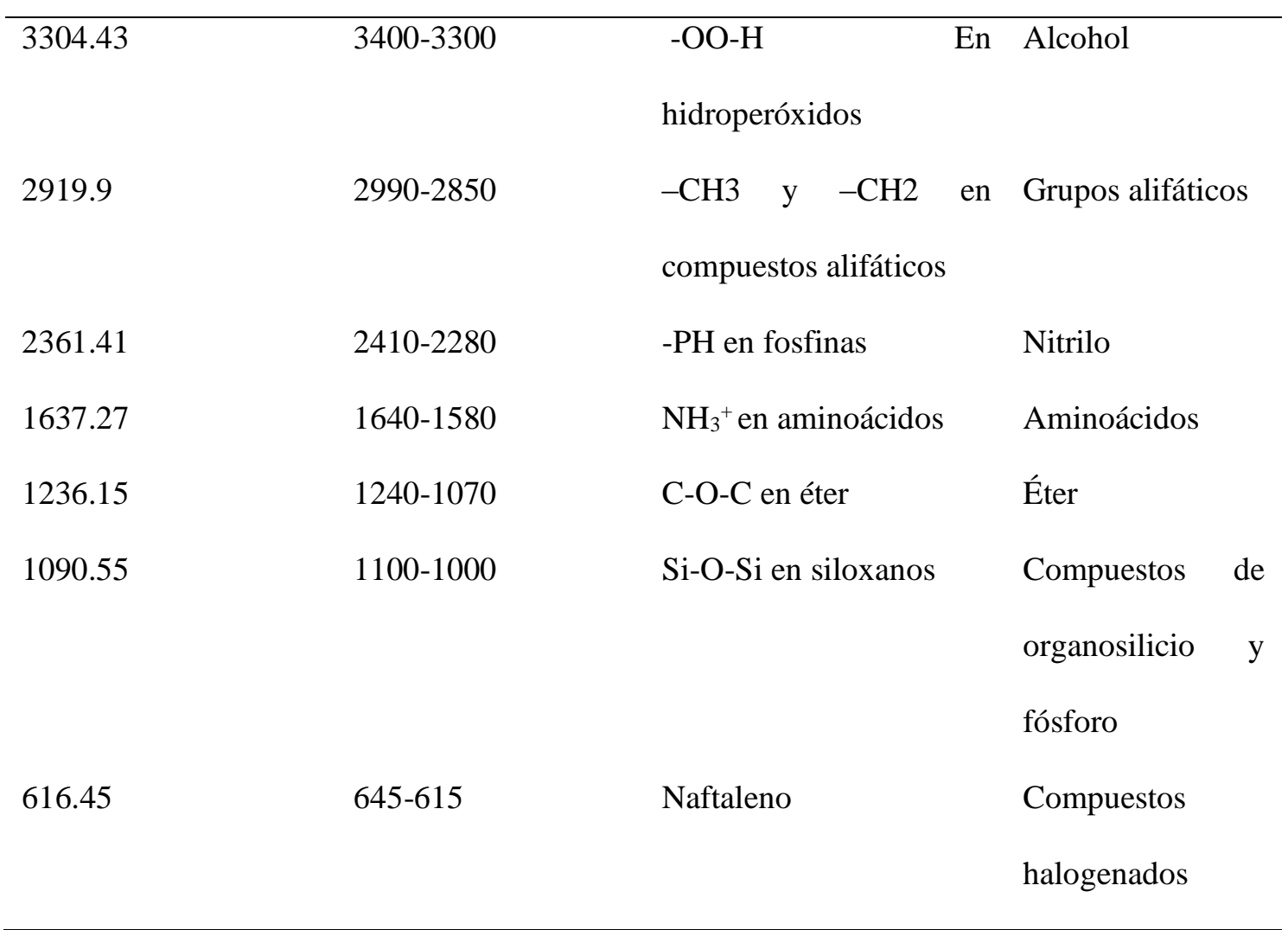

Fuente: Spectra-Structure Correlations in the Mid- and Far-infrared. H.F. Shurvell. Queen's University, Kingston, Ontario, Canada.

\section{Discusión}

La investigación confirma la aplicación de una eficiente tecnología desarrollada para la obtención de vinagre de bambú, por la Facultad de Ciencia y Tecnología de Materiales de la Universidad Forestal de Beijing (china) y que ha sido adaptada a nuestro medio potenciando el uso de residuos orgánicos disponibles y accesibles, en este contexto, por su naturaleza y origen, contribuye a la gestión de impactos ambientales negativos significativos del medio y con posteriores investigaciones, una potencial contribución con el ambiente, reduciendo la utilización de productos químicos como fertilizantes que a largo plazo, inhiben los ciclos biológicos de los suelos para su regeneración natural. Con los análisis realizados al producto, es posible relacionarlo con el denominado vinagre de bambú, cuyos componentes identificados fueron del 70,65\% en base al área del pico de espectros, en la que los componentes acéticos ocuparon el 42,32\%, con el ácido acético como componente principal, los componentes fenólicos del 23,22\% con fenol, guaiacol y su syringol como componentes principales, Componentes neutros del 5,11\% con furfural y ciclopentanona. (Jun, 2006), El vinagre de bambú, actualmente es utilizado para potenciar el crecimiento radicular, con los porcentajes que se menciona. El análisis que arroja el FTIR, son similares al vinagre de bambú, se puede asumir, que el vinagre proveniente de $P$. clandestinum $L$, podría ser usado con éxito para crecimiento radicular entre otras aplicaciones agropecuarias que serían probadas con futuras investigaciones. 
Una posible valoración económica de los residuos orgánicos producto de diferentes actividades de poda no han sido de interés de los sistemas económicos por tratarse de material desechado, es necesario incluir a este proceso en el marco de la economía, su inserción a la actividad económica como materia primaria para su reutilización se vería determinada por la posible utilidad agropecuaria de los subproductos como reemplazo de géneros comerciales que cumplen las mismas funciones pero reduciendo los riesgos ambientales por su naturaleza orgánica. (Aguilera, 2006).

\section{Conclusiones}

- Como subproducto de la pirolisis de la materia orgánica se obtiene el biocarbón, en una cantidad de $17 \mathrm{~kg}$ de una biomasa inicial de $42 \mathrm{~kg}$, es decir, con $40 \%$ de rendimiento, el cual debido a sus características físicas es propicio para sistemas de secuestro de $\mathrm{CO}_{2}$.

- $\quad$ El análisis FTIR del destilado obtenido del proceso realizado evidencia la presencia de compuestos orgánicos como: ácidos carboxílicos, fenoles, cetonas y aldehídos con lo que se confirma la similitud con los productos obtenidos de procesos realizados para el bambú.

- El proceso de destilación no operó al $100 \%$ de su capacidad debido a errores sistemáticos y aleatorios por lo que se obtuvo $500 \mathrm{ml}$ de ácido piroleñoso, la optimización de sistema aumenta la posibilidad de obtener mayores volúmenes de ácido piroleñoso.

- La eficiencia del proceso depende especialmente de la cantidad de ácido acético obtenido, pues es el principio activo más utilizado, se determina que el rendimiento varía mucho con la clase y mezcla de residuos utilizados en el proceso, a más de la eficiencia de condensación.

- Si el kikuyo cuenta con un elevado promedio de fracción soluble de proteína cruda (Tabla 1), no es posible que exista cantidades de proteína en el condensado obtenido, sin embargo, al existir concentraciones de fracciones no degradables permite suponer que exista también en el proceso de destilado, se considera también la composición inicial en base seca para determinar la eficiencia del producto aplicado en el suelo.

- La acentuada crisis económica, la cultura productiva, la dependencia de los productores de la fertilización química, los bajos niveles productivos y el deterioro constante de los suelos evidencia la importancia del uso de esta tecnología como una alternativa productiva de bajo costo y con énfasis agroecológico.

\section{Agradecimientos}

A los señores estudiantes Alex Escobar, Byron Cajamarca, Félix Vaca y Diego Carrillo de la carrera de Ingeniería en Biotecnología Ambiental de la Escuela 
Superior Politécnica de Chimborazo, que fueron parte fundamental de la presente investigación.

\section{Referencias Bibliográficas}

Aguilera, U. (2006). El valor económico del medio ambiente. Obtenido de:

http://www.revistaecosistemas.net/artículo

Bradley, M. (2002). Logotipo de Thermo Fisher Scientific. Obtenido de Fundamentos de FTIR: https://www.thermofisher.com/ec/en/home/industrial/spectroscopyelemental-isotope-analysis/spectroscopy-elemental-isotope-analysis-learningcenter/molecular-spectroscopy-information/ftir-information/ftir-basics.html

Burnette, R. (2013). An Introduction to Wood Vinager. Obtenido de c.ymcdn.com: https://c.ymcdn.com/sites/echocommunity.siteym.com/resource/collection/D9D576A1-771A-4D95-A8894FBD9E75D03D/TN_77_An_Introduction_to_Wood_Vinegar.pdf

CharMarker. (2009). Producing Pyrolysis Liquids and wood vinegar from biomass. Obtenido de www.esenergy.com.au: http://www.esenergy.com.au/wpcontent/uploads/2016/03/CharMaker-MPP-Pyrolysis-liquids-production.pdf

Choi, J. Y. (2009). Effect of Wood Vinegar on the Performance, Nutrient Digestibility and Intestinal Microflora in Weanling Pigs. Asian-Aust. J. Anim. Sci., 8.

Hagner, M. (2013). Potential of the slow pyrolysis products ISBN 978-952-10-9169-8 . Obtenido de helda.helsinki.fi: https://helda.helsinki.fi/bitstream/handle/10138/40305/hagner_dissertation.pdf

Ikeshima, Y. (1999). Method of Producing and Using Bamboo Charcoal and Bamboo Vinegar. Nosan Gyoson Bunka Kyokai, 23.

Jun, M. (2006). Preliminary study of application effect of bamboo vinegar on vegetable growth. Stud. China, 5. 


\section{PARA CITAR EL ARTÍCULO INDEXADO.}

Beltrán Dávalos, A., Escudero Vilema, M., Córdova Morales, S., \& Rosero Erazo, C. (2019).

Monitoreo microbiológico para la gestión ambiental de Aguas Residuales Hospitalarias. Ciencia Digital, 3(3.4.), 242-253. https://doi.org/10.33262/cienciadigital.v3i3.4.882

\section{Ciencia \\ LDigital}

El artículo que se publica es de exclusiva responsabilidad de los autores y no necesariamente reflejan el pensamiento de la Revista Ciencia Digital.

El artículo queda en propiedad de la revista y, por tanto, su publicación parcial y/o total en otro medio tiene que ser autorizado por el director de la Revista Ciencia Digital.
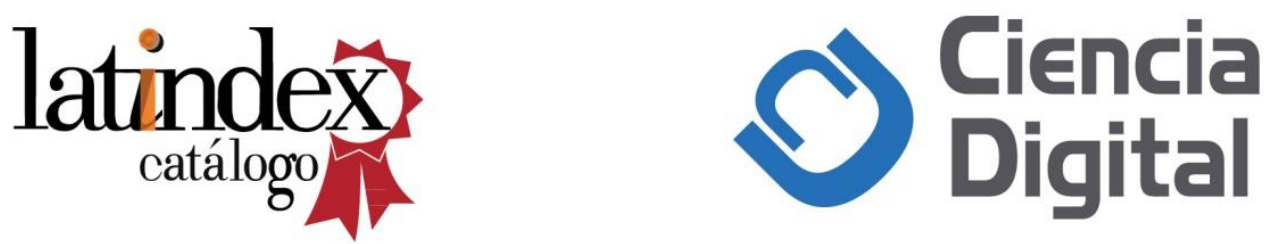